\title{
Development of Hodgkin lymphoma in a patient with sarcoidosis
}

\author{
F. Gediz', A.F. Yilmaz'1, B. Payzin'1, T. Yuksel'1, A.O. Calli², S. Kobak ${ }^{3}$ \\ ${ }^{1}$ Department of Hematology, Katip Celebi University, Ataturk Education and Research Hospital, Izmir, Turkey; \\ 2Department of Pathology, Katip Celebi University, Ataturk Education and Research Hospital, Izmir, Turkey; \\ ${ }^{3}$ Department of Rheumatology, Istinye University, Faculty of Medicine, LIV Hospital, Istanbul, Turkey
}

\section{SUMMARY}

Sarcoidosis is a chronic granulomatous disease of unknown etiology characterized by non-caseified granulomas in many different organs and systems. The disease most frequently manifests with bilateral hilar lymphadenopathy and infiltrations in the lungs and skin, as well as with eye lesions. It may mimic a number of systemic diseases and/or accompany them. The development of lymphoma in patients with sarcoidosis or the co-occurrence of both diseases is rarely reported in the literature. In this paper we report a female patient followed up with sarcoidosis for three years who developed Hodgkin lymphoma, according to the results of the investigations and biopsy results.

Key words: Sarcoidosis; Hodgkin lymphoma; Development.

\section{INTRODUCTION}

arcoidosis is a systemic disease of un$\checkmark$ known etiology that is characterized by non-caseified granuloma reaction that can involve multiple organs (1). Although its pathogenesis is not clear, there appears to be a cellular immune system activation and a non-specific inflammatory response in the presence of some genetic and environmental factors. Th1 cells and macrophages stimulated by pro-inflammatory cytokines induce the inflammatory cascade and the formation of granulomas occur as a result of tissue permeability, cellular influx and local cell proliferation (2). The crucial pathological evidence of sarcoidosis is non-caseified epithelioid cellular granulomas. Epithelioid cells are transformed bone marrow monocytes that have prominent seretory activity. An increased number of active CD4 T lymphocytes are shown in sarcoidosis tissues. While these lymphocytes diffusely increase in the granulomatous lesion, smaller quantities of CD-8 T-Ly, B-cells, plasma and mast cells are found in the periphery of the granuloma. The fact that the disease shows differing prevalence, clinical findings and disease courses in different race and ethnical groups is an indicator that sarcoidosis is a heterogeneous disease (3). It is generally seen in women and it appears at the $4^{\text {th }}$ decade. While the incidence of sarcoidosis in the white race of the USA is 10.9 in 100,000 , this rate increases to 35.5 in Afro-Americans in whom the progress of the disease is more severe (4). Sarcoidosis is a chronic inflammatory disease that can manifest with various clinical findings. Chronic inflammation may be a risk factor for the development of a malignancy and malignancy rates in sarcoidosis patients were reported as $1.2-2.5 \%$ (5). Lung cancer, lymphoma and uterus cancers are frequently seen (6). This paper reports the co-occurrence of sarcoidosis and Hodgkin lymphoma, which is very rare.

\section{CASE REPORT}

A 39-year-old female patient presented to our Hematology clinic with swellings on her neck and groin, fever, weight loss and night sweating. Her medical history revealed that she had been investigated 
and followed up in the Rheumatology outpatient clinic from the year 2012. On the basis of recurrent uveitis attacks and bilateral hilar lymphadenopathies detected on torax computed tomography (CT), she was suspected for sarcoidosis and the lymph node biopsy taken by endobronchial ultrasonography (EBUS) method revealed non-caseified granuloma formation (Figure 1). Conditions that might cause granulomatous disease (such as bacterial and fungal infections, and lymphoma) had been ruled out and the patient had been diagnosed as sarcoidosis stage 1 according to the Scadding criteria. Because she had recurrent uveitis attacks, she had occasionally been treated with prednisone $20 \mathrm{mg} /$ day. Three years later, the patient suffered from new complaints such as fever, weight loss and night sweating and was referred to our Hematology clinic. At physical examination, bilateral cervical lymph nodes $(7.5 \mathrm{x}$ $6.5 \mathrm{~mm}$ ) and three lymph nodes of $2 \mathrm{~cm}$ in here left groin were palpated. There were no organomegalies. Her laboratory examination results were: $\mathrm{Hgb}: 12.4 \mathrm{~g} / \mathrm{L}, \mathrm{MCV}$ : 81, WBC: 27.100, Neu: 7710, Neu\%28.5, Lym\%62, Plt: 396000, Vitamin B12: 469 ng/L, Ferritin: 106 ng/mL.

Liver functions and kidney functions were normal. The acute phase reactants were: $\mathrm{C}$ reactive protein (CRP): $5.38 \mathrm{mg} / \mathrm{dl}$, erythrocyte sedimentation rate (ESR): $29 \mathrm{~mm} / \mathrm{h}$. The level of serum angiotensin converting enzyme (ACE) was $86 \mathrm{mg} / \mathrm{dL}$ (normal $<45$ $\mathrm{mg} / \mathrm{dL}$ ). There was polyclonal gammopathy in her protein electrophoresis. Bilateral hilar and mediastinal lymphadenopathies were present in her thorax CT (Figure 2). Even if she had been followed up because of sarcoidosis, besides cervical and inguinal lymphadenopathies, she had complaints such as weight loss and night sweating as well, which is why we performed a lymph node biopsy.

The lymph node was taken from the left cervical region and led to diagnosis of mixed cellular type Hodgkin lymphoma with markers; CD15 (+), CD30 (-), CD20 (-), CD45 (-) (Figure 3). A bone marrow biopsy was performed for staging which revealed no infiltration. The patient was evaluated by FDG positron emission tomography associated with computed tomography (PET/CT) for staging. Bilateral cervical, hilar, mediastinal, para tracheal, paracaval, iliac, inguinal lymphadenopathies and sclerotic changes in the bone were found on PET-CT and the disease was staged as 3B mixed cellular Hodgkin lymphoma. The patient was started with ABVD (doxorubicin, bleomycin, vinblastine, and dacarbazine) chemotherapy regimen treatment and scheduled for follow up.

The PET/CT that was performed after the fourth course of the treatment revealed the

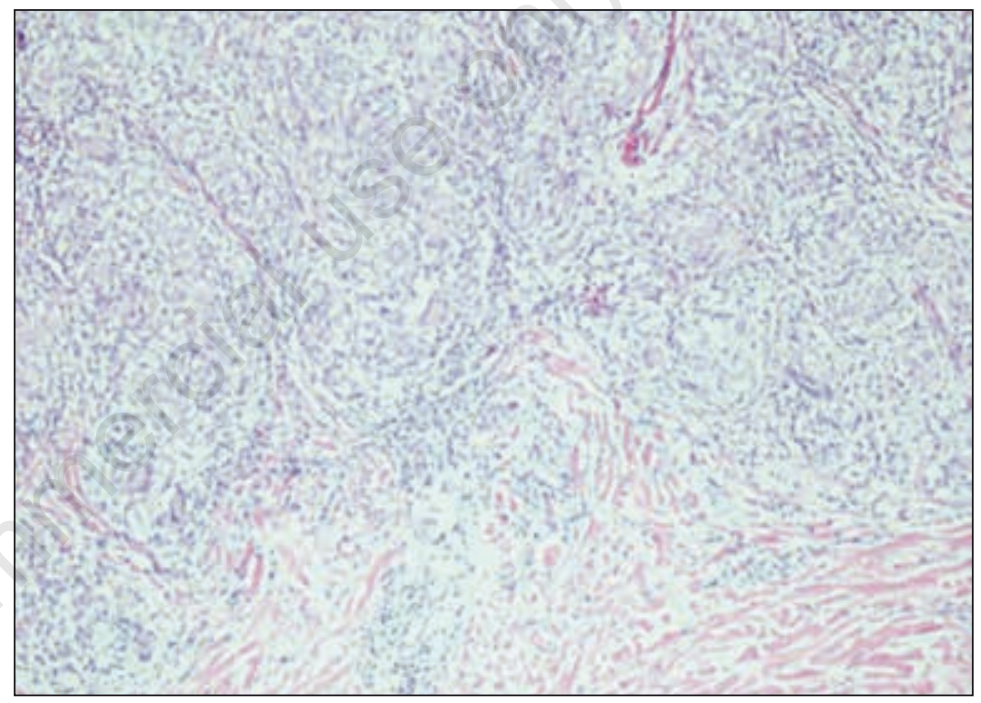

Figure 1 - Lymph node biopsy revealed non-caseified granuloma formation.

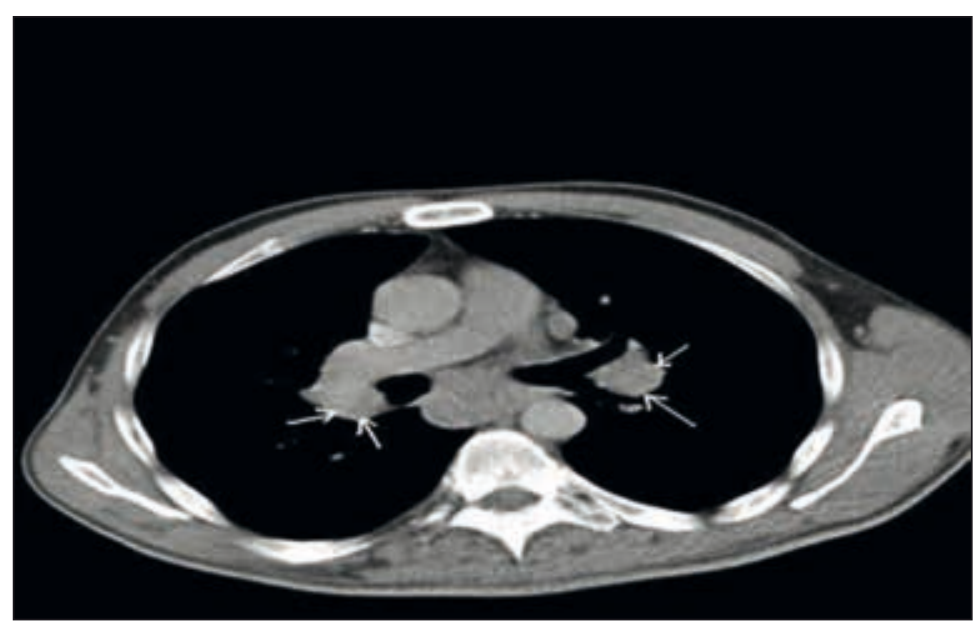

Figure 2 - Thorax CT showing bilateral hilar and mediastinal lymphadenopaties. 


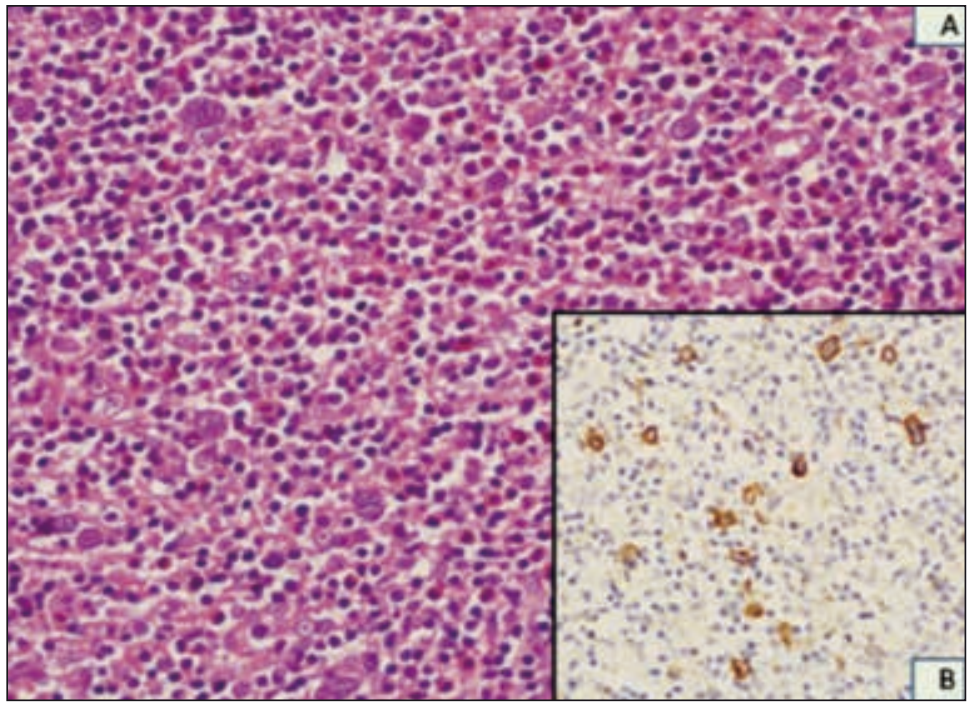

Figure 3 - A) Figure showing mixed cellular population in Hodgkin lymphoma with various inflammatory cells, Reed-Stenberg cells (H\&Ex220). B) Reed-Stenberg and Hodgkin cells selectively immunostained for CD-30 marker (x110).

regression of nearly all the lymphadenopathies. The follow-up and the treatment of the patient, who remains clinically stable, is still in progress.

\section{DISCUSSION AND CONCLUSIONS}

In this paper, we report a patient who has been followed up for sarcoidosis and was subsequently diagnosed with Hodgkin lymphoma as a result of examinations performed because of increasing complaints. The initial diagnosis of sarcoidosis had been made by the rheumatologists with reference to clinical indications and symptoms and histopathological confirmation of non-caseified granulomas on lymph node biopsy. The risk of malignancy development (especially lymphomas and lung cancer) is increased in patients with sarcoidosis. Chronic inflammation is an important risk for the carcinogenesis process. Sarcoidosis-lymphoma syndrome was first described by Brinker (7). The etiopathogenesis of this co-occurrence may be explained by immunological abnormalities seen in several malignancies and lymphoproliferative disorders.

Sarcoidosis and lymphoma co-occurrence may be detected simultaneously, but generally lymphoma develops one or two years later. In our case, the development of lymphoma from a sarcoidosis base took nearly three years. Patients with sarcoidosis have a high risk of development of malignant lymphoma, and this association was defined with the term sarcoidosis-lymphoma syndrome. Sarcoidal granulomas can be observed in patients with malignant tumors, including malignant lymphoma and carcinoma without history of systemic sarcoidosis. Ishida et al. reported a 60-year-old Japanese female patient with extranodal marginal zone lymphoma of mucosa-associated lymphoid tissue (MALT lymphoma) who developed sarcoidosis two years after treatment (8).

Goswami et al. reported 4 patients diagnosed with sarcoidosis either during or after the treatment of lymphoma (9). Although rare, there are previous case reports about the co-occurrence of sarcoidosis and Hodgkin disease. Silva et al. reported sarcoidosis diagnosed with biopsy in a 37-year-old female patient appearing with erythema nodosum and bilateral hilar lymphadenopathies (10). Three years later, this patient manifested widespread lymphadenopathies and was diagnosed with mixed type Hodgkin lymphoma. However, the development of sarcoidosis in patients with lymphoma was reported too. London et al. reported sarcoidosis in 39 lymphoma (19 Hodgkin) patients (11). Mean development duration was 18 months and only 16 of the patients were treated. Complete remission was provided in $98 \%$ of the treated patients. Sarcoidosis is a chronic inflammatory disease characterized by non-caseified granulomas and lymphoid hyperplasia resulting in the dysfunction of the immunoregulatory pathways. There are many immunological similarities between sarcoidosis and lymphoma; both have cutaneous anergy, peripheral lymphadenopathies and over infiltration of $\mathrm{T}$ helper cells in tissues. According to one theory, systemic sarcoidosis is initiated by the cell-mediated immune response that develops against the tumor antigens that were secreted by the non-caseified granu- 
loma inside the tumor (12). It is important to recognize that systemic sarcoidosis and sarcoidal reaction without evidence of systemic disease can occur after development of malignant lymphoma. Therefore, sarcoidal reaction must be included in the differential diagnostic consideration of recurrent malignant lymphoma.

In conclusion, we reported the development of Hodgkin lymphoma in a patient followed up for a diagnosis of sarcoidosis. Sarcoidosis and lymphoma co-occurrence mainly consists of the development of lymphoma after sarcoidosis. Even rare sarcoidosis may develop in patients with lymphoma. Since clinical manifestations of both diseases are similar, especially if new findings such as fever, weight loss, splenomegaly and new lymphadenopathies appear, histolopathological confirmation is needed. New prospective studies into the pathogenesis of the sarcoidosis-lymphoma syndrome are necessary.

\section{REFERENCES}

1. Newman LS, Rose CS, Maier LA. Sarcoidosis. N Engl J Med. 1997; 336: 1224-34.

2. Hofmann S, Franke A, Fischer A, et al. Genome-wide association study identifies
ANXA11 as a new susceptibility locus for sarcoidosis. Nat Genet. 2008; 40: 1103-6.

3. Chen ES, Moller DR. Etiology of sarcoidosis. Clin Chest Med. 2008; 29: 365-77.

4. Smith G, Brownell I, Sanchez M, Prystowsky $\mathrm{S}$. Advances in the genetics of sarcoidosis. Clin Genet. 2008; 73: 401-12.

5. Askling J, Grunewald J, Eklund A, et al. Increased risk for cancer following sarcoidosis. Am J Respir Crit Care Med. 1999; 160: 166872.

6. Cohen PR, Kurzrock R. Sarcoidosis and malignancy. Clin Dermatol. 2007; 25: 326-33.

7. Brincker H. The sarcoidosis-lymphoma syndrome. Br J Cancer. 1986; 54: 467-73.

8. Ishida M, Hodohara K, Furuya A, et al. Sarcoidal granulomas in the mediastinal lymph nodes after treatment for marginal zone lymphoma of the esophagus: report of a case with review of the concept of the sarcoidosis-lymphoma syndrome. Int J Clin Exp Pathol. 2014; 7: 4428-32.

9. Goswami T, Siddique S, Cohen P, Cheson BD. The sarcoid-lymphoma syndrome. Clin Lymphoma Myeloma Leuk. 2010; 10: 241-7.

10. Silva GN, Morais MF, Raposo J, et al. Hodgkin's disease in a patient with sarcoidosis. Acta Med Portuguesa. 1996; 9: 287-93.

11. London J, Grados A, Fermé C, et al. Sarcoidosis occurring after lymphoma: report of 14 patients and review of the literature. Medicine (Baltimore). 2014; 93: e121.

12. Maayan H, Ashkenazi Y, Nagler A, et al. Sarcoidosis and lymphoma: case series and literature review. Sarcoidosis Vasc Diffuse Lung Dis. 2011; 28: 146-52. 